\title{
LOS DETERMINANTES DE LA SATISFACCIÓN LABORAL DE LOS TITULADOS DE ECONOMÍA Y EMPRESA
}

\author{
Cristina Borra Marcos* \\ Francisco Gómez García*1, Manuel Salas Velasco ${ }^{\dagger}$, \\ *Universidad de Sevilla, †Universidad de Granada
}

Recibido el 26 de febrero de 2007; aceptado el 10 de octubre de 2007

\section{Resumen}

Este trabajo examina los determinantes de la satisfacción laboral de una cohorte de jóvenes graduados: los que obtuvieron su titulación en el curso 2001/2002 en las carreras del área económica de la Universidad de Sevilla. Tras estimar un logit ordenado se obtiene la conclusión que en la evaluación que realizan los encuestados sobre su grado de satisfacción en el trabajo actual los individuos valoran recompensas tanto monetarias o extrínsecas (salario) como no monetarias o intrínsecas (poder realizarse en el trabajo, posibilidad de conciliación trabajo/familia).

Clasificación JEL: J28, J31.

Palabras clave: Satisfacción laboral, educación superior, modelo logit ordenado.

\section{Abstract}

This paper examines job satisfaction determinants of a cohort of young graduates: those that obtained their degree in Business, Management, Economics or Marketing in the academic year 2001/2002 at the University of Seville. We estimate an ordered logit model and conclude that, in evaluating job satisfaction, individuals consider both monetary or extrinsic gains (wage) and non-monetary or intrinsic gains (personal accomplishment, work/family reconciliation).

JEL Classification: J28, J31.

Key words: Job satisfaction, higher education, ordered logit model.

\footnotetext{
1 Autor para correspondencia: fgomez@us.es. Agradecemos la financiación del proyecto en que se basa este estudio por parte del Centro de Estudios Andaluces (ECOD2.05/038). Estamos especialmente agradecidos a José Ignacio García, responsable de seguimiento de dicho proyecto. Asimismo, agradecemos la excelente asistencia de investigación por parte de Margarita Mariscal. Por último, también agradecemos los comentarios de un evaluador anónimo y del editor de la Revista de Economía Laboral. Los errores que persistan son de nuestra exclusiva responsabilidad.
}

(C) Revista de Economía Laboral 


\section{Introducción}

En nuestra investigación abordamos el estudio del grado de satisfacción que manifiestan los graduados universitarios en relación con algunos aspectos del puesto de trabajo actual que ellos desempeñan, pues esto determina, entre otras cuestiones, sus intenciones de permanencia o no en la empresa -véase, por ejemplo, Freeman (1978), Clark et al. (1998) y Lévy-Garboua et al. (2001)-. Además la satisfacción laboral está relacionada con: a) el absentismo (Kenyon y Dawkins, 1989; Johansson y Palme, 1996); b) la productividad (Mangione y Quinn, 1975) y c) la propia salud del trabajador (Bacharach et al., 1991).

Nuestro trabajo se basa en la muestra generada en una investigación, cuyo objetivo principal es analizar los determinantes de la inserción y de la satisfacción laboral de un grupo concreto de graduados: los que han cursado carreras del área económica en la Universidad de Sevilla (diplomado en Empresariales, licenciado en Economía, licenciado en Administración y Dirección de Empresas y licenciado en Investigación y Técnicas de Mercado). Por tanto, el estudio se implementa a partir de una cohorte de jóvenes graduados ${ }^{2}$.

Aunque la mayoría de los autores centran su estudio en la relación entre la satisfacción laboral y una variable en particular (salario, género, etc.), nosotros hemos realizado un estudio de tipo general, donde se incluyen ciertas variables (como la "conciliación") que no han solido incluirse en los estudios sobre satisfacción laboral. Además, como es habitual, trabajamos con datos de corte transversal. Para contextualizar el interés de este trabajo, hay que tener en cuenta la observación de Teichler (2003) sobre la escasez de las fuentes de información sobre la relación entre la educación superior y el mundo laboral.

En el apartado segundo se realiza una breve descripción estadística de la muestra que sirve de soporte del análisis econométrico posterior. En el apartado tercero se implementa el análisis microeconométrico de la satisfacción laboral de los economistas incluidos en nuestra muestra. Por último, el apartado cuarto recoge las principales conclusiones de nuestra investigación.

\section{Descripción de la base de datos}

El estudio utiliza datos de carácter primario obtenidos mediante una encuesta realizada a egresados de la Diplomatura de Empresariales y

\footnotetext{
2 Mora y Ferrer-i-Carbonell (2006) también realizan un trabajo sobre satisfacción laboral con una cohorte de jóvenes graduados de Cataluña.
} 
de las Licenciaturas de Economía, Administración de Empresas y Marketing de la Universidad de Sevilla. Con el objetivo de tener una perspectiva -trayectoria laboral- de cuatro años, se seleccionaron aquellos egresados que terminaron sus estudios en el curso académico 2001/2002. Se envió el cuestionario por correo postal a la totalidad de la población (982 individuos) y se obtuvo una tasa de respuesta del 21 por ciento, una tasa aceptable teniendo en cuenta la extensión del cuestionario ${ }^{3}$, donde además de la satisfacción se engloban cuestiones sobre la duración del primer desempleo, el ajuste educación-empleo, las trayectorias laborales, los niveles salariales y, entre otras, las características socio-demográficas y académicas de los egresados ${ }^{4}$.

La encuesta nos ha permitido reconstruir la historia académica y laboral de estos graduados. El Cuadro 1 muestra la comparación entre la muestra y la población objetivo. En líneas generales, la similitud de dichas distribuciones en el caso de algunas características de los egresados (sexo, edad, titulación ${ }^{5}$ ) confirma la representatividad de la muestra.

Cuadro 1. Comparación de la muestra y la población de estudio.

\begin{tabular}{lcc}
\hline & Muestra & Población \\
\hline Diplom. Empresariales & $50 \%$ & $62 \%$ \\
Lic. Economía & $25 \%$ & $12 \%$ \\
LADE & $16 \%$ & $17 \%$ \\
Lic. Marketing & $9 \%$ & $9 \%$ \\
Sexo (=1 Hombre) & $43 \%$ & $40,1 \%$ \\
Edad media & 28,86 & 29,2 \\
\hline
\end{tabular}

Fuente: Elaboración propia a partir de los datos suministrados por las secretarías del campus de Economía (Universidad de Sevilla)

En el cuestionario se pidió a los egresados que expresaran su grado de satisfacción general en el puesto de trabajo y con relación a distintos aspectos como el sueldo, la seguridad en el puesto, las posibilidades de ascenso, el clima laboral o el tipo de liderazgo ejercido. Nuestra variable dependiente es el grado de satisfacción global en el trabajo medido subjetivamente en una escala entre 1 (muy insatisfecho) y 5 (muy insatisfecho). El Cuadro 2 presenta la distribución, general y por sexos, del grado de satisfacción global en el trabajo actual de los individuos de la muestra.

\footnotetext{
${ }^{3}$ Disponible a petición de los interesados.

${ }^{4}$ El trabajo de campo tuvo lugar durante el año 2006.

${ }^{5}$ En el caso de los diplomados en Empresariales y los licenciados en Economía existe un intercambio de porcentajes en la muestra en relación a la población.
} 
Cuadro 2. Grado de satisfacción global por sexo

\begin{tabular}{lrrr}
\hline \multirow{2}{*}{ Grado de satisfacción } & \multicolumn{2}{c}{ Sexo } & \multirow{2}{*}{ Total } \\
\cline { 2 - 3 } & Hombre & Mujer & \\
\hline Muy insatisfecho & - & $3,2 \%$ & $1,8 \%$ \\
Insatisfecho & $5,8 \%$ & $5,3 \%$ & $5,5 \%$ \\
Satisfecho & $23,2 \%$ & $20,2 \%$ & $21,5 \%$ \\
Bastante Satisfecho & $53,6 \%$ & $45,7 \%$ & $49,1 \%$ \\
Muy Satisfecho & $17,4 \%$ & $25,5 \%$ & $22,1 \%$ \\
\hline Total & $100,0 \%$ & $100,0 \%$ & $100,0 \%$ \\
\hline
\end{tabular}

Fuente: Elaboración propia.

Como se observa, pese a que hombres y mujeres alcanzan prácticamente un mismo nivel de satisfacción media, las mujeres presentan una distribución mucho más dispersa. Hay más mujeres muy insatisfechas y también más mujeres muy satisfechas con su trabajo.

Respecto a los resultados totales -el 71,2 por ciento de los egresados está bastante o muy satisfecho con su empleo actual-, la distribución de la satisfacción laboral observada en el Cuadro 2 es consistente con estudios realizados en otros países, como el Reino Unido, que muestran que los niveles revelados de satisfacción por parte de los trabajadores son muy altos (Millward et al., 1999) ${ }^{6}$. Esos resultados pueden reflejar un efecto de auto-selección, en el sentido de que los trabajadores eligen trabajos que les gustan y abandonan aquellos que no les gustan.

\section{Análisis microeconométrico de la satisfacción laboral}

A partir de la literatura especializada podemos esperar que la satisfacción laboral (SL) dependa del siguiente grupo de variables ${ }^{7}$ :

\footnotetext{
${ }^{6}$ Para el caso español, una de las características más significativas de los resultados de la Encuesta de Calidad de Vida en el Trabajo (ECVT)-que elabora el Ministerio de Trabajo y Asuntos Sociales- para el período 2000-2004 es la elevada calificación que los trabajadores otorgan a la satisfacción global sobre su trabajo (Ripoll et al., 2006).

${ }^{7}$ En Gamero (2005) puede encontrarse una excelente sistematización de este cuerpo de literatura.
} 
$\mathrm{SL}=\mathrm{f}$ (características personales, tipo de carrera, características objetivas del empleo, valoración subjetiva de los intangibles del puesto de trabajo, otras variables)

Con la finalidad de conocer qué factores o variables son fuente de mayor satisfacción y cuáles de menor satisfacción entre los encuestados (en relación con su trabajo actual) recurrimos al análisis econométrico. Para tener en cuenta la naturaleza discreta y ordenada de la variable dependiente -el grado de satisfacción laboral global- se especifica un modelo logit ordenado (Greene, 1999):

$$
Y^{*}=\beta^{\prime} X+\varepsilon
$$

donde $\varepsilon$ es la perturbación, que sigue una distribución logística, $X$ es el vector de regresores -incluida la constante- que recoge características observables de los individuos encuestados (edad, estado civil, etc.) y, por último, el vector de coeficientes beta cuantifica el impacto de los regresores utilizados. No obstante, la variable $Y^{*}$ (variable latente continua) no la podemos observar; lo que observamos es:

$$
\begin{array}{ccc}
Y=0 & \text { si } & Y^{*} \leq 0 \\
Y=1 & \text { si } & 0<Y^{*} \leq \mu_{1} \\
Y=2 & \text { si } & \mu_{1}<Y^{*} \leq \mu_{2} \\
Y=3 & \text { si } & \mu_{2}<Y^{*} \leq \mu_{3} \\
\cdots \cdots \cdots . . & & \\
Y=J & \text { si } & Y^{*}>\mu_{j-1}
\end{array}
$$

Los coeficientes mu (puntos de umbral o barrera) son parámetros que se han de estimar al tiempo que las betas. La variable $Y$, que es una variable observada (intensidad de la satisfacción), toma los siguientes valores en nuestro caso: $Y=0$ si el encuestado da una valoración de 1, 2 ó 3 (satisfacción baja); $Y=1$ si el encuestado da una valoración de 4 (satisfacción media); y $Y=2$ si el encuestado da una valoración de 5 (satisfacción alta). El encuestado, al responder sobre el nivel de satisfacción laboral, puede escoger entre estos valores, dependiendo de si se considera desde muy insatisfecho hasta muy satisfecho ${ }^{8}$.

8 La variable dependiente es la satisfacción global en el empleo actual (variable cualitativa politómica construida a partir de la información proporcionada por la 
Las variables independientes incluidas en el análisis son: el salario; el género; el estado civil y la edad; un conjunto de variables dicotómicas que reflejan el título universitario alcanzado; variables relacionadas con el puesto de trabajo (cargo, tipo de contrato, etc.); y un grupo de indicadores que miden la discrepancia entre lo que un titulado espera de un trabajo y la realidad de su empleo en relación con aspectos como, por ejemplo, la realización personal.

El Cuadro 3 muestra los resultados de la estimación del modelo logit ordenado para la satisfacción laboral global especificado anteriormente. Se han estimado tres modelos: el modelo 1, que es el modelo más completo; y los modelos 2 y 3 , que excluyen algunas variables explicativas para evitar la multicolinealidad ${ }^{9}$. En el modelo logit ordenado si el coeficiente estimado asociado a una variable es positivo $y$ estadísticamente significativo nos indica que la variable en cuestión es un factor que aumenta la satisfacción laboral. Por su parte, valores negativos y estadísticamente significativos se asocian con factores que reducen la satisfacción laboral ${ }^{10}$.

Los resultados -modelo 1- confirman que en la evaluación que realizan los encuestados sobre su grado de satisfacción en el trabajo actual los individuos valoran recompensas tanto monetarias como no monetarias.

El salario es la característica del empleo que mayor interés ha suscitado en los economistas preocupados por los determinantes de la satisfacción laboral. Desde un punto de vista teórico la conexión parece clara. Sin embargo, la evidencia en relación con esta cuestión es mixta. Groot y Brink (1999a) no descubren efecto significativo, mientras que Clark y Oswald (1996) encuentran que el nivel de satisfacción declarado disminuye con el salario. Por otro lado, Sloane y Williams (2000) hallan correlación positiva para ambos sexos. Groot y Brink (1999b) proponen una explicación basada en el cambio de las preferencias del trabajador con el nivel de ganancias (el efecto del incremento salarial sobre la satisfacción laboral acaba evaporándose). Nuestro resultado, al encontrar que el salario es una de las principales fuentes de satisfacción de los egresados, es coherente, pues trabajamos con una muestra de trabajadores jóvenes.

pregunta correspondiente -pregunta 27, apartado a, columna B). En concreto se realizó la pregunta: "¿Cuál es tu grado de satisfacción global en el trabajo actual? Valora de 1 (muy insatisfecho) a 5 (muy satisfecho)...”. La agrupación realizada obedece al reducido número de casos en las categorías 1 y 2 de la encuesta.

9 Por ejemplo, el estado civil está altamente correlacionado con la edad (correlación igual a 0,357 ) y el tipo de contrato con el salario (correlación igual a 0,339).

10 Preferimos decir "reducen la satisfacción" en lugar de "aumentan la insatisfacción" porque son muy pocos los individuos que afirman sentirse insatisfechos o muy insatisfechos con su trabajo actual. 
Sin embargo, también encontramos evidencia de que el no poder realizarse en el trabajo, y el no poder combinar empleo y familia11, constituyen factores que reducen la satisfacción de los titulados con sus empleos. La variable "poder realizarse en el trabajo" es estadísticamente significativa y esto claramente refleja la importancia de las características intangibles del empleo. No sólo el salario es importante, también lo son las relaciones personales $\mathrm{y}$, entre otras cuestiones, la misión de la organización en la que se trabaja. Además, la variable "conciliación" es otra característica intangible que hasta ahora no ha solido incluirse en la literatura y que nosotros encontramos bastante significativa.

Por titulaciones, se encuentra que los niveles de educación más elevados se asocian con trabajadores menos satisfechos: en concreto los licenciados en Económicas están menos contentos o satisfechos con sus empleos que los diplomados en Empresariales (categoría de referencia) ${ }^{12}$. Esto puede estar poniendo de manifiesto que quienes han estudiado Economía tienen más dificultades para encontrar un trabajo acorde con su formación, lo que puede terminar generando cierta frustración.

En el modelo 2 (que se centra en las características objetivas del empleo), podemos observar que por puestos de responsabilidad, los mandos intermedios están más satisfechos con su trabajo, todo lo demás constante, que administrativos o comerciales (categoría de referencia); resultado previsible, ya que estos últimos son puestos más rutinarios y van acompañados normalmente de menores niveles de responsabilidad, iniciativa y capacidad para tomar decisiones. Nuestro resultado está en consonancia con los resultados obtenidos por Lydon y Chevalier (2002) y Belfield y Harris (2002) para el Reino Unido ${ }^{13}$.

11 En la pregunta 28 del cuestionario se preguntó a los graduados: “Cómo de importantes son para ti las siguientes características de una ocupación? En la columna $A$ da tus valoraciones personales en general; en la $B$ señala lo importantes que son en tu trabajo actual. Valora de 1 (nada importante) a 5 (muy importante)...". Así, las variables "discrepancia en relación con la realización personal" y de "conciliación" se incluyen como continuas y toman valores comprendidos entre -4 (mínima discrepancia) y +4 (máxima discrepancia).

${ }^{12}$ Los diplomados en Empresariales son la categoría de referencia, al estar altamente correlacionada con la edad (correlación igual a 0.300; estadísticamente significativa al 1 por ciento). Los licenciados en Administración de Empresas y en Marketing también están menos satisfechos que los diplomados, pero los coeficientes respectivos no son significativos.

13 Se observa que, cuando no se controlan por las características personales y académicas de los egresados -modelo 2-, son fuentes de satisfacción: a) trabajar en el sector financiero y b) trabajar en el sector público. 


\section{Cuadro 3. Modelo logit ordenado de satisfacción global en} el empleo actual.

\begin{tabular}{|c|c|c|c|c|c|c|c|c|c|c|c|}
\hline \multirow[b]{2}{*}{ Variable } & \multicolumn{3}{|c|}{ Modelo 1} & \multicolumn{3}{|c|}{ Modelo 2} & \multicolumn{3}{|c|}{ Modelo 3} & \multirow[b]{2}{*}{ Med $\ddagger$} & \multirow[b]{2}{*}{ D.t. } \\
\hline & Coef. & & $t$ & Coef. & & $t$ & Coef. & & $t$ & & \\
\hline Constante & $-10,763$ & $*$ & $-1,87$ & 1,119 & & 1,41 & $-11,728$ & $* *$ & $-2,81$ & & \\
\hline Edad (en años) & $-0,008$ & & $-0,92$ & & & & $-0,113$ & & $-1,62$ & 28,86 & 2,74 \\
\hline Género (= 1 hombre) & $-0,646$ & & $-1,57$ & & & & $-0,649$ & * & $-1,73$ & 0,43 & \\
\hline Estado Civil (= 1 casado) & 0,405 & & 0,81 & & & & & & & 0,23 & \\
\hline \multicolumn{12}{|l|}{ Titulación: } \\
\hline Dipl. Empresariales (Ref.) & - & & - & & & & - & & - & 0,50 & \\
\hline Lic. Economía & $-1,621$ & $* *$ & $-2,99$ & & & & $-1,448$ & $* *$ & $-2,87$ & 0,25 & \\
\hline $\mathrm{LADE}^{\dagger}$ & $-0,280$ & & $-0,48$ & & & & $-0,085$ & & $-0,16$ & 0,16 & \\
\hline Lic. Marketing & $-0,326$ & & $-0,40$ & & & & $-0,160$ & & $-0,25$ & 0,009 & \\
\hline $\begin{array}{l}\text { Discrepancia con realización } \\
\text { personal }\end{array}$ & $-0,838$ & $* *$ & $-3,93$ & & & & $-0,939$ & $* *$ & $-4,70$ & 1,02 & 1,06 \\
\hline $\begin{array}{l}\text { Discrepancia con conciliación } \\
\text { familiar }\end{array}$ & $-0,336$ & $* *$ & $-2,14$ & & & & $-0,332$ & $* *$ & $-2,33$ & 1,22 & 1,38 \\
\hline Salario (en logs) & 2,334 & $* *$ & 3,27 & & & & 2,599 & $* *$ & 4,55 & 7,08 & 0,38 \\
\hline \multicolumn{12}{|l|}{ Tipo de puesto: } \\
\hline Responsabilidad baja & 0,211 & & 0,31 & 0,398 & & 0,69 & & & & 0,45 & \\
\hline Comerciales (Ref.) & - & & - & - & & - & & & & 0,12 & \\
\hline Responsabilidad media-alta & 0,615 & & 0,97 & 1,157 & $* *$ & 1,97 & & & & 0,43 & \\
\hline Tipo de contrato (= 1 fijo) & 0,300 & & 0,66 & & & & & & & 0,70 & \\
\hline Tipo de empresa (= 1 privada) & $-0,778$ & & $-1,10$ & $-1,554$ & $* *$ & $-2,50$ & & & & 0,90 & \\
\hline \multicolumn{12}{|l|}{ Actividad de la empresa: } \\
\hline Sector financiero & 0,890 & & 1,54 & 1,826 & $* *$ & 3,85 & & & & 0,30 & \\
\hline Construcción/industria & 0,188 & & 0,31 & 0,664 & & 1,28 & & & & 0,18 & \\
\hline Consultoría & 0,904 & & 1,39 & 0,837 & & 1,49 & & & & 0,12 & \\
\hline Otra (Ref.) & - & & & - & & & & & & 0,40 & \\
\hline$\mu(1)$ & 3,373 & $* *$ & 8,74 & 2,635 & $* *$ & 9,34 & 3,202 & $* *$ & 8,967 & & \\
\hline Log función verosimilitud & $-107,71$ & & & $-129,59$ & & & $-111,30$ & & & & \\
\hline Chi 2 & 67,930 & $* *$ & & 24,163 & $* *$ & & 60,744 & $* *$ & & & \\
\hline
\end{tabular}

Por género -véase modelo 3 , donde no se controla por las características objetivas del empleo, excepto por el salario-, y en línea con los trabajos sobre satisfacción (véase, por ejemplo, Clark, 1997), los hombres están menos satisfechos con sus empleos que las mujeres -hay que indicar que se ha encontrado sólo un nivel de significación del 10 por 
ciento para la variable género-. Algunos investigadores han puesto el énfasis en que esas discrepancias en satisfacción son el resultado de los distintos esquemas valorativos respecto a las características de los empleos (Groot y Brink, 1998; Sloane y Williams, 2000). Si efectivamente las mujeres valoran más las características intangibles de su trabajo (por ejemplo, relaciones personales) podrían estar, ceteris paribus, más satisfechas con sus trabajos ${ }^{14}$.

Por último, hay que indicar que la evidencia sobre el impacto de la eventualidad de la relación contractual sobre la satisfacción es mixta. Clark y Oswald (1996) encuentran el efecto positivo esperado de poseer un contrato indefinido, mientras en Clark (1996) la relación no resulta estadísticamente significativa. Nosotros -modelo 1-, aunque el tener un contrato indefinido aumenta la satisfacción de los encuestados, tampoco hemos encontrado una relación estadísticamente significativa. Una posible explicación es que la satisfacción puede depender del tipo de contrato (temporal o indefinido), aunque la relación es esencialmente indirecta, operando a través del salario -véase Penard et al. (1999).

\section{Conclusiones}

El interés de nuestra profesión por cuestiones como la felicidad o la satisfacción laboral es relativamente reciente. En nuestro trabajo analizamos los determinantes de la satisfacción laboral de un grupo concreto de jóvenes graduados: los economistas que terminaron sus estudios en el curso 2001/2002 en la Universidad de Sevilla. Dada la inexistencia de investigaciones estadísticas oficiales que recopilen la información apropiada, nuestra investigación se ha sustentado en una base de datos elaborada dentro de nuestro propio equipo de investigación, a partir de un cuestionario piloto validado convenientemente. Aunque con las cautelas pertinentes, nuestro análisis empírico implementado a partir de dicha base de datos sugiere las siguientes conclusiones.

Los resultados confirman que en la evaluación que realizan los encuestados sobre su grado de satisfacción en el trabajo actual los individuos valoran recompensas tanto monetarias como no monetarias. Así, se constata que el salario es una de las fuentes principales de

\footnotetext{
14 Sin embargo, cuando controlamos por todas las características objetivas del empleo modelo 1-, desaparece este efecto diferencial por género. En este punto hay que tener en cuenta que, como se observa en el Cuadro 2, un 3,2 por ciento de las mujeres de la muestra se sienten muy insatisfechas con sus trabajos actuales (porcentaje nulo para los hombres). Aquí podríamos encontrar indicios de discriminación laboral, aunque este análisis excede el ámbito de nuestra investigación.
} 
satisfacción laboral. Otra fuente importante, vinculada a la anterior, es ser mando intermedio.

Pero también son importantes las características intangibles del empleo, como el no poder realizarse en el trabajo, y el no poder conciliar empleo y familia, factores que reducen la satisfacción de los titulados con sus empleos. Por tanto, características individuales que han recibido poca atención en la literatura, como los valores laborales, muestran capacidad predictiva sobre la satisfacción laboral global.

\section{Bibliografía}

Bacharach, S.B., Bamberger, P. y Conley, S. (1991): "Work-home conflict among Nurses and Engineers: Mediating the Impact of Role Stress on Burnout and Satisfaction at Work", Journal of Organizational Behavior, 12, pp. 39-53.

Belfield, C.R. y Harris, R.D.F. (2002): "How Well do Theories of Job Matching Explain Variations in Job Satisfaction across Education Levels? Evidence for UK Graduates", Applied Economics, 34, pp. 535548 .

Clark, A.E. (1996): "Job Satisfaction in Britain", British Journal of Industrial Relations, 34, pp. 189-217.

Clark, A.E. (1997): "Job Satisfaction and Gender: Why Are Women so Happy at Work?", Labour Economics, 4, pp. 341-372.

Clark, A. E. y Oswald, A.J. (1996): "Satisfaction and Comparison Income", Journal of Public Economics, 61, pp. 359-381.

Clarl, A.E., Georgellis, Y. y Sanfey, P. (1998): "Job Satisfaction, Wage Changes and Quits: Evidence from Germany", Research in Labor Economics, 17, pp. 95-121.

Freeman, R. B. (1978): "Job Satisfaction as an Economic Variable", American Economic Review, Papers and Proceedings, 68, pp. 135-141.

Gamero, C. (2005): Análisis Microeconómico de la Satisfacción Laboral, Consejo Económico y Social, Colección Estudios nº 171. 
Greene, W.H. (1999): Análisis Econométrico, Prentice Hall Iberia, Madrid.

Groot, W. y Brink, M.V.D. (1998): "Job Satisfaction, Wages and Allocation of Men and Women”, TSER/STT Working Papers, WP, pp. 10-98.

Groot, W. y Brink, M.V.D. (1999a): "Job Satisfaction of Older Workers", International Journal of Manpower, 20, pp. 343-360.

Groot, W. y Brink, M.V.D. (1999b): “Job Satisfaction and Preference Drift”, Economic Letters, 63, pp. 363-367.

Johansson, P. y Palme, M. (1996): "Do Economic Incentives Affect Work Absence? Empirical Evidence Using Swedish Micro Data", Journal of Public Economics, 59, pp. 195-218.

Kenyon, P. y Dawkins, P. (1989): "A Time Series Analysis of Labour Absence in Australia", Review of Economics and Statistics, 71, pp. 232239.

Lévy-Garboua, L., Montmarquette, C. y Simonnet, V. (2001): “Job Satisfaction and Quits: Theory and Evidence from the German Socioeconomic Panel", CIRANO Working Papers, Scientific Series, 2001s-41.

Lydon, R. y Chevalier, R. (2002): "Estimates of the Effect of Wages on Job Satisfaction”, CEP-LSE Discussion Papers, 0531.

Mangione, T.W. y Quinn, R.P. (1975): "Job Satisfaction, Counterproductive Behaviour and Drug Use at Work", Journal of Applied Psychology, 60, pp. 114-116.

Millward, N. Forth, J. y Bryson, A. (1999): All Change at Work?, Routledge, London.

Mora, T. y Ferrer-i-Carbonell, A. (2006): "The Job Satisfaction Gender Gap among Young Recent University Graduates: Evidence from Catalonia”, XXXI Simposio de Análisis Económico, Oviedo.

Peñard, R. Sollogoub, M. y Ulrich, V. (1999): “The Integration of Youths into the Labour Market and the Type of Employment Contract: An 
Approach Based on the Theory of Repeated Games", TSER/STT Working Papers, WP, pp. 30-99.

Ripoll, E. Falguera, M. y Urrutia, J. (2006): "Factores determinantes de la satisfacción en el trabajo: Cinco años del barómetro laboral”, Índice, enero, pp. 14-16.

Sloane, P.J. y Williams, H. (2000): "Job Satisfaction, Comparison Earnings and Gender”, Labour, 14, pp. 473-501.

Teichler, U. (2003): "Aspectos Metodológicos de las Encuestas a Graduados Universitarios”, en Vidal, J. (coord.): Métodos de Análisis de la Inserción Laboral de los Universitarios, Consejo de Coordinación Universitaria, Ministerio de Educación, Cultura y Deporte, pp. 15-29. 